\title{
BMJ Open What is the influence of single-entry models on access to elective surgical procedures? A systematic review
}

\author{
Zaheed Damani, Barbara Conner-Spady, Tina Nash, Henry Tom Stelfox, \\ Tom W Noseworthy, Deborah A Marshall
}

To cite: Damani Z, ConnerSpady B, Nash T, et al. What is the influence of singleentry models on access to elective surgical procedures? A systematic review. BMJ Open 2017;6:e012225. doi:10.1136/bmjopen-2016012225

- Prepublication history and additional material is available. To view please visit the journal (http://dx.doi.org/ 10.1136/bmjopen-2016012225).

Received 14 April 2016 Revised 12 December 2016 Accepted 14 December 2016

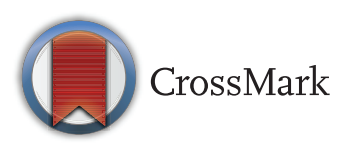

Department of Community Health Sciences, Cumming School of Medicine, University of Calgary, Calgary, Alberta, Canada

Correspondence to Dr Deborah A Marshall; damarsha@ucalgary.ca

\section{ABSTRACT}

Background: Single-entry models (SEMs) for the management of patients awaiting elective surgical services are designed to increase access and flow through the system of care. We assessed scope of use and influence of SEMs on access (waiting times/ throughput) and patient-centredness (patient/provider acceptability).

Methods: Systematic review of articles published in 6 relevant electronic databases included studies from database inception to July 2016. Included studies needed to (1) report on the nature of the SEM; (2) specify elective service and (3) address at least 1 of 3 research questions related to (1) scope of use of SEMs; (2) influence on timeliness and access; (3) patient-centredness and acceptability. Article quality was assessed using a modified Downs and Black checklist.

Results: 11 studies from Canada, Australia and the UK were included with mostly weak observational design-2 simulations, 5 before-after, 2 descriptive and 2 cross-sectional studies. 9 studies showed a decrease in patient waiting times; 6 showed that more patients were meeting benchmark waiting times; and 5 demonstrated that waiting lists decreased using an SEM as compared with controls. Patient acceptability was examined in 6 studies, with high levels of satisfaction reported. Acceptability among general practitioners/surgeons was mixed, as reported in 1 study. Research varied widely in design, scope, reported outcomes and overall quality.

Conclusions: This is the first review to assess the influence of SEMs on access to elective surgery for adults. This review demonstrates a potential ability for SEMs to improve timeliness and patient-centredness of elective services; however, the small number of lowquality studies available does not support firm conclusions about the effectiveness of SEMs to improve access. Further evaluation with higher quality designs and rigour is required.

\section{INTRODUCTION}

Long waiting times for elective healthcare services pose complex clinical, organisational, economic and political issues,

\section{Strengths and limitations of this study}

- Our article is the first systematic review on single-entry models (SEMs) for adult elective surgical services and the first to examine and summarise the influence of SEMs on patient flow, waiting times and acceptability.

- Using a semiquantitative representation explicitly identifies gaps in the existing literature and highlights areas where further research will be essential in strengthening the overall understanding of influence of SEMs on domains of healthcare quality beyond access, as defined by the Institute of Medicine (IOM).

- Very few studies exist in the literature that evaluates the influence of SEMs on timeliness and patient-centredness (patient and provider acceptability).

- The literature is of varying quality (mostly weak observational design) and small in overall quantity and consequently, it is difficult to establish that using an SEM causes improvement in the quality of care-more rigourous studies are needed.

- Grey literature was not reviewed.

especially in nations with universal healthcare systems. ${ }^{1}$ Restricting access and long waiting times serve as tools for rationing in these systems, where demand for services often exceeds the available supply of resources. ${ }^{2}$ Expectations and demand for greater efficiency continues to grow as populations are ageing, living longer and are increasingly accessing elective procedures, leading to deterioration in overall access to care. ${ }^{13}$ Waiting beyond a certain amount of time can be harmful for health, generate dissatisfaction and can lead to deterioration in income and public confidence in the healthcare system. ${ }^{4}$ There are several management strategies available to improve access and reduce waiting times for elective surgical services. The study of waiting time management strategies ${ }^{14-11}$ has shown that simply adding 
more resources is not effective. ${ }^{1}{ }^{3}$ Changes in service provision (through structures and programmes) combined with improvements in management are being recommended as part of novel approaches to address these issues. ${ }^{1}{ }^{12}$ Single-entry models (SEMs) are one such approach.

Single entry is a commonly used waiting time management strategy in the airline, banking and restaurant industries whereby customers assemble in a single queue to see the next-available service provider. Waiting time variability and the average wait can be reduced. ${ }^{13}$ SEMs are increasingly being used in healthcare and can consist of several components: pooled/common waiting lists (consolidation of multiple waiting lists); centralised intake (a single point-of-entry through which referrals are received and service provision arranged) and triage (through which referrals are assessed for appropriateness and/or urgency). ${ }^{14}$ The necessary component in SEMs is pooling for the purposes of service provision-it creates a single list of patients and pools service providers (clinicians) so that patients can see the next one available. With all three components of SEMs present, patients access services through a single point-of-entry and are able to see the next-available provider on a firstcome, first-served basis (priority and urgency assessed in some cases). ${ }^{5}$ In theory, this improves the distribution and flow of patients throughout the system. Delays and time lost to waiting, as compared with when there are multiple lines, are reduced because a single line continues to be serviced-patients seeing the next-available physician (from a pool of participating physicians) means no longer having to wait for an appointment slot for a specific physician to become available. ${ }^{14}$

No systematic reviews have assessed the influence of SEMs on access to elective surgical services. Growing evidence suggests that single entry is an important management strategy that should be more broadly applied in healthcare to reduce waiting times and increase patient flow. ${ }^{12} 15 \quad 16$ However, this evidence has neither been compiled nor carefully examined, albeit it has major policy implications for improving patient access across clinical services and health systems. This review examines SEMs using the domains of quality related to 'access' and as defined by the Institute for Medicine: timeliness (reducing waits and sometimes harmful delays) and patient-centeredness (providing care that is respectful of and responsive to individual patient preferences, needs, and values and ensuring that patient values guide all clinical decisions). ${ }^{17}$ The work is driven by a central research question, supplemented by three supporting questions:

What is the influence of SEMs on access to elective surgical services for adults?

A. Where are SEMs used in healthcare and how are they implemented?

B. What is the influence of SEMs on timeliness of elective surgical services?

C. To what extent are SEMs acceptable to patients and acceptable to providers?
The objective of this paper is to review and summarise existing research evidence on the scope, use and implementation of SEMs for elective surgical services, specifically with respect to the influence of SEMs on patient flow and waiting times for elective procedures in adults and acceptability of SEMs to patients and providers (general practitioners (GPs) and surgeons).

\section{METHODS}

\section{Criteria for considering eligible studies}

Original studies and empiric works were included if they involved adult human participants ( $\geq 18$ years) and had to do with both elective surgical services and waiting time management. Studies needed to (1) report on components of single entry used; (2) focus on elective service type and (3) address at least one of our three supporting research questions. All study designs were included in an effort to be comprehensive. Review articles, commentaries, case reports and studies that featured non-adult participants ( $<18$ years), involved animals, featured no elements of SEMs, reported on non-elective services (emergent, transplant, mental health or long-term care), did not report on waiting times or were non-English were excluded.

\section{Search strategy}

We used MeSH headings and terms related to single entry (central intake, pooling, triage), timeliness (wait list, queue) and elective surgical services (elective/ routine service, surgery, scheduled services; see online supplementary appendix 1). Type of elective service was not constrained, allowing for broadest inclusion. Timeliness was measured in terms of qualitative, quantitative and/or proportional reporting on waiting list length, waiting times, benchmark targets, changes in patient volume, efficiency of care provision and equity of access. ${ }^{17} 18$ Patient-centredness was defined as patient, physician and/or surgeon satisfaction and acceptability of care. $^{17}{ }^{18}$ MEDLINE, EMBASE, CINAHL, the Cochrane Database for Systematic Reviews, CENTRAL (Cochrane Central Registry of Controlled Trials) and Abstract Business Information (ABI)/Inform were initially searched to locate peerreviewed literature, using all languages, from inception of the database until July 2016. The reference lists of included articles were also scanned for additional relevant references.

\section{Screening and data analysis}

Two reviewers completed article screening and data abstraction in two stages-title and abstract followed by full-text review-using a standard, pilot-tested template. Discrepancies were resolved by consensus. For each study, we analysed: purpose, clinical area/procedure represented, country of origin, model of single-entry employed (intervention), comparator, study design, data source, sample source, sample size and relevant primary 
outcome variables. The influence of SEMs on timeliness and patient-centredness of elective services were extracted and summarised narratively and semiquantitatively, in a systematic fashion using tabular form. Study results and limitations were also summarised. Owing to heterogeneity in study design, population and definitions/primary outcomes, meta-analysis was not considered and therefore a narrative synthesis approach was used. ${ }^{19}$ This review and approach adheres to PRISMA criteria for reporting systematic reviews that evaluate healthcare interventions. ${ }^{20}$

\section{Quality assessment}

A 21-item quality assessment guide was adapted from the Downs and Black checklist ${ }^{21}$ to assess the overall quality of included studies. The tool is easy to use, provides an overall numerical score for study quality and has been assessed to be valid and reliable. ${ }^{22}$ While there are several risk of bias assessment tools available for nonrandomised studies, the Downs and Black checklist is frequently recommended and among the few developed for use in systematic reviews. ${ }^{23}$ Quality of reporting, internal and external validity, appropriateness of methods, validity of authors' conclusions and overall clarity/presentation were rated to enable comparison between studies. The adapted checklist was piloted and administered by the two raters. Items were scored from 0 to 1 , and a score of 0.5 was given in some instances. Non-applicable items were not reflected in the score. A quality index (QI) was calculated to generate comparable aggregate scores for each study. A total score of 0-21 (best) was derived by multiplying the average value of applicable items by 21 . The mean score of the two raters' quality assessments was rounded to the nearest whole number. Ten key indicators that were applicable and comparable across all studies were extracted for reporting in this review. Items were dichotomously scored as 'yes' or 'no', based on their reporting.

\section{RESULTS}

\section{Study selection}

A total of 3672 citations were identified. Sixty-two full studies were reviewed, and 11 were selected for final analysis (see figure 1 for PRISMA flow diagram) - 2 discrete event simulation trials, 5 before-after studies, 2

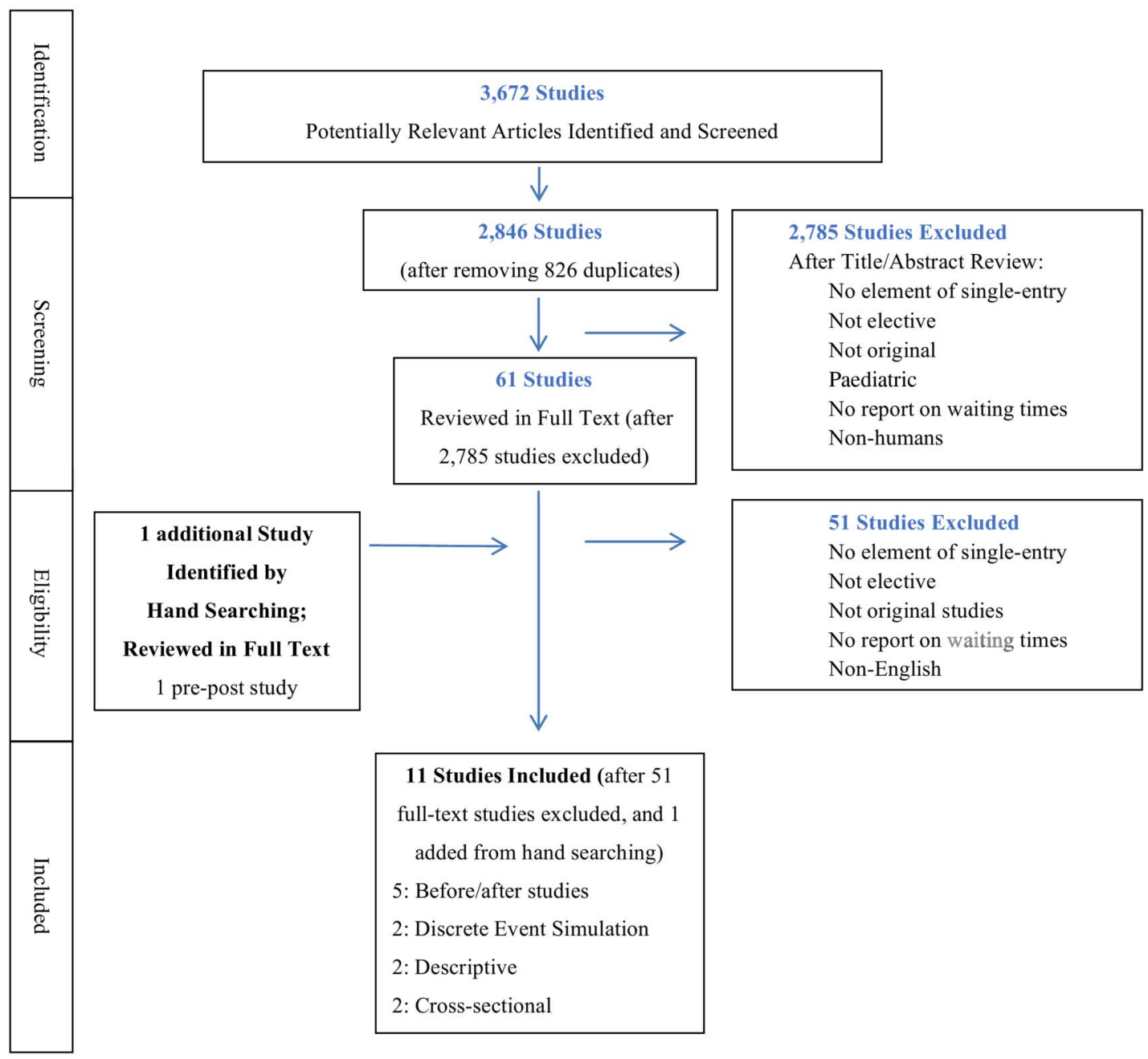

Figure 1 PRISMA flow diagram of study inclusion. 
descriptive studies and 2 cross-sectional studies. Lack of elements related to single entry and no report on waiting times/waiting lists were the most common reasons for exclusion. All discrepancies were decided by consensus.

\section{Article characteristics and quality}

Table 1 summarises the characteristics of included studies. Included studies were published from 2002 to 2012. Seven studies were from Canada, ${ }^{24-30}$ three from UK and one from Australia. ${ }^{31}$ Three studies evaluated the use of SEMs for cardiac surgery, two for hernia, two for hip and knee replacement, one for spinal surgery, one for cataract removal and two studies for multiple elective procedures. With the exception of one, ${ }^{26}$ all studies were evaluation studies that used quasiexperimental or weak observational designs.

Table 2 summarises the results and limitations of the included studies. The scope/use of SEMs to some extent (research question 1) was addressed by all 11 studies; the influence of SEMs on timeliness (research question 2) by 9 and the influence of SEMs on patientcentredness and provider acceptability (research question 3) by 5 .

Table 3 summarises study quality, with QI scores ranging from 9 to 20 (mean 14); higher scores indicate greater rigour in the methods and reporting of results (based on the Downs and Black checklist ${ }^{21}$ ). Included studies were generally of low rigour, with limited data, analysis and reporting on few outcomes.

\section{The use of SEMs in healthcare systems}

Eight studies reported the use of SEMs for surgery ${ }^{5}$ 27-33 (including two discrete event simulation studies, which modelled their use); three for outpatient surgical service consultations. ${ }^{24-26}$ Six detailed the implementation and effectiveness of SEMs, ${ }^{24} 2528$ 31-33 two simulated the outcomes of a potential implementation, ${ }^{27}{ }^{30}$ two described the development of such an implementation ${ }^{26} 28$ and two assessed the views of physicians and/or patients regarding the use of SEMs. ${ }^{5} 29$

Pooled waiting lists, centralised intake and triage are the most common components of single entry, often implemented in combination. ${ }^{29}$ Services employing centralised intake generally resulted in improved distribution of procedures across service areas. ${ }^{29} 31$ Triage reduced waiting times for the most severe patients. ${ }^{24} 32$ Triage involves administrative and clinical staff, including nurses, ${ }^{29} 31{ }^{32}$ physiotherapists, ${ }^{28}$ pharmacists ${ }^{25}{ }^{26}$ and consultants $^{33}$ to determine priority and suitability for care while reducing delays, unnecessary appointments and work for specialists. ${ }^{31}$ Two studies employed pooled lists only, ${ }^{5} 27$ the remaining nine studies employed all three components ${ }^{24-26}$ 28-31 33 (including one simulation study).$^{29}$ The components of single entry and their characteristics are described in online supplementary appendix 2.

\section{Influence of SEMs on timeliness of elective services}

To improve timeliness, most studies combined components of single entry with tools to assess appropriateness, determine priority ${ }^{24-26} 3133$ or conduct comprehensive patient preassessment. Studies used various indicators to measure changes in timeliness. Waiting times were measured in three ways: (1) from referral to consultation (WT1); (2) consultation to surgery (WT2); (3) referral to surgery (the sum of WT1 and WT2). Table 4 summarises the study results and outcomes.

Four studies (including one simulation study) ${ }^{27} 30$ reported a decrease in waiting list length (based on the number of patients waiting), ${ }^{30-33}$ when compared with a control group or to historic experience of controls. With the exception of one study where waiting lists were eliminated, ${ }^{31}$ magnitude is difficult to assess, as the remaining three studies describe reductions in waiting list length qualitatively, as a trend.

All nine studies assessing waiting times showed some measure of reduced waiting times (including both simulation studies) $;{ }^{27}$ however, there was a lack of uniformity and consistency in reporting across studies. Studies reported WT1, WT2 or total waiting time, but rarely reported all three. Without knowing both WT1 and WT2, it is difficult to conclude whether overall patient waiting times are reduced. For cardiac consultation, Bungard $e t a l^{25}{ }^{26}$ demonstrated a reduction in both. For cardiac surgery, Vasilakis et $a l^{30}$ using a discrete event simulation, demonstrated a differential impact on WT1 and WT2 and across surgical priority groups. These differences are critical to evaluate, especially from a public policy perspective. Three studies (including one simulation) ${ }^{29}$ reported that waiting times decreased for those who had been waiting longest and increased slightly for less urgent cases; $;^{25} 3033$ however, these were reported generally as trends, rather than with specific quantitative data. In two studies, this was reported only graphically $^{30} 33$ without any supporting explanation.

Six studies (including both simulation studies) ${ }^{27} 30$ demonstrated that SEMs increased the proportion of individuals meeting clinically recommended benchmark waiting times. ${ }^{25} 2728303233$ There was lack of consistency here also-target benchmarks were related to either WT1 or WT2 and varied depending on the procedure or jurisdiction.

Three studies suggested that with SEMs the number of patient referrals increased, allowing for expanded patient throughput (volume). ${ }^{24} \quad 25 \quad 31 \quad 32$ Five studies demonstrated an increase in the appropriateness of referrals being received (reductions in inappropriate referrals where patients do not meet the developed criteria). ${ }^{24} 25283233$ Given the complexity of interventions, it is difficult to attribute these improvements to specific components of single entry.

Six studies (including one simulation) reported that SEMs improved efficiency of patient management; ${ }^{24} 2527293132$ and three (including one simulation) reported a reduction in variation of waiting list 
Table 1 Study characteristics

\begin{tabular}{|c|c|c|c|c|c|c|c|c|}
\hline $\begin{array}{l}\text { Author/year/ } \\
\text { country }\end{array}$ & Intervention & $\begin{array}{l}\text { Research } \\
\text { question } \\
\text { addressed }\end{array}$ & Study design & Sample source & Sample size & $\begin{array}{l}\text { Model of } \\
\text { single } \\
\text { entry }\end{array}$ & Data source & $\begin{array}{l}\text { Primary outcome } \\
\text { measures }\end{array}$ \\
\hline $\begin{array}{l}\text { Ramchandani } \\
\text { et al }(2002)^{5} / \\
\text { UK }\end{array}$ & $\begin{array}{l}\text { Pooled waiting lists for } \\
\text { cataract surgery, whereby } \\
\text { Pts are treated in turn by } \\
\text { the first available surgeon }\end{array}$ & $\begin{array}{l}\text { Scope of use } \\
\text { Acceptability }\end{array}$ & Cross-sectional & $\begin{array}{l}\text { Cataract Pts, GPs } \\
\text { and ophthalmologists } \\
\text { in Birmingham }\end{array}$ & $\begin{array}{l}85 \text { Pts } \\
50 \text { GPs } \\
479 \\
\text { ophthalmologists }\end{array}$ & $P$ & $\begin{array}{l}\text { Questionnaire } \\
\text { Interview }\end{array}$ & $\begin{array}{l}\text { Opinion on } \\
\text { pooled waiting } \\
\text { lists }\end{array}$ \\
\hline $\begin{array}{l}\text { Leach et al } \\
(2004)^{33} / \mathrm{UK}\end{array}$ & $\begin{array}{l}\text { Two systems to reduce } \\
\text { WTs for elective } \\
\text { non-complex spinal } \\
\text { surgery: generic booking } \\
\text { system for apts/surgery } \\
\text { and MRI booking system }\end{array}$ & $\begin{array}{l}\text { Scope of use } \\
\text { Timeliness } \\
\text { Acceptability }\end{array}$ & $\begin{array}{l}\text { Simple pre-post } \\
\text { with non- } \\
\text { equivalent } \\
\text { groups }\end{array}$ & $\begin{array}{l}\text { Pts in Manchester } \\
\text { awaiting } \\
\text { non-complex spinal } \\
\text { surgery }\end{array}$ & Not reported & $\mathrm{P}, \mathrm{C}, \mathrm{T}$ & $\begin{array}{l}\text { Administrative } \\
\text { data }\end{array}$ & $\begin{array}{l}\text { WT1: referral to } \\
\text { first apt } \\
\text { WT2: scan to } \\
\text { outpatient } \\
\text { review } \\
\text { Time on waiting } \\
\text { list for surgery } \\
\text { (>3/6/9 months) }\end{array}$ \\
\hline $\begin{array}{l}\text { Singh et al } \\
(2005)^{31} / \\
\text { Australia }\end{array}$ & $\begin{array}{l}\text { Pooled regional surgical } \\
\text { referrals and altered } \\
\text { procedure for surgical } \\
\text { admission (use of a new } \\
\text { booking and waiting list } \\
\text { system administered by a } \\
\text { coordinator; restructured } \\
\text { surgical operating } \\
\text { sessions; postdischarge } \\
\text { model clinical pathway) }\end{array}$ & $\begin{array}{l}\text { Scope of use } \\
\text { Timeliness } \\
\text { Acceptability }\end{array}$ & $\begin{array}{l}\text { Post-test only } \\
\text { with non- } \\
\text { equivalent } \\
\text { groups }\end{array}$ & $\begin{array}{l}\text { Various elective } \\
\text { procedures- } \\
\text { Western Sydney } \\
\text { Area Health Service }\end{array}$ & $\begin{array}{l}12 \text { surgeons } \\
143 \mathrm{Pts}\end{array}$ & $P, C, T$ & $\begin{array}{l}\text { Administrative } \\
\text { Data } \\
\text { Questionnaire }\end{array}$ & $\begin{array}{l}\text { Number of } \\
\text { procedures } \\
\text { performed } \\
\text { Operating time/ } \\
\text { discharge rate/ } \\
\text { length of stay } \\
\text { Waiting list } \\
\text { length } \\
\text { Operating costs } \\
\text { - Pt satisfaction }\end{array}$ \\
\hline $\begin{array}{l}\text { Sri-Ram et al } \\
(2005)^{32} / \mathrm{UK}\end{array}$ & $\begin{array}{l}\text { Offering Pts a direct } \\
\text { booking service, triage by } \\
\text { surgeon, nurse-led } \\
\text { preassessment in the day } \\
\text { surgery unit; fit Pts offered } \\
\text { an appointment within } \\
4 \text { weeks }\end{array}$ & $\begin{array}{l}\text { Scope of use } \\
\text { Timeliness } \\
\text { Acceptability }\end{array}$ & $\begin{array}{l}\text { Post-test only } \\
\text { with non- } \\
\text { equivalent } \\
\text { groups }\end{array}$ & $\begin{array}{l}\text { All inguinal hernia } \\
\text { services-the } \\
\text { Whittington Hospital } \\
\text { (London) }\end{array}$ & $\begin{array}{l}\text { Intervention: } 74 \\
\text { Pts Control: } 147 \\
\text { Pts }\end{array}$ & $P, C, T$ & $\begin{array}{l}\text { Administrative } \\
\text { Data } \\
\text { Questionnaire }\end{array}$ & $\begin{array}{l}\text { WT1: referral to } \\
\text { first apt } \\
\text { WT2: from apt } \\
\text { to surg } \\
\text { Total WT } \\
\text { Tumber of } \\
\text { procedures } \\
\text { performed } \\
\text { Pt acceptability }\end{array}$ \\
\hline $\begin{array}{l}\text { Vasilakis et al } \\
(2007)^{30} / \\
\text { Canada }\end{array}$ & $\begin{array}{l}\text { Discrete event simulation } \\
\text { to compare and assess } \\
\text { two methods of scheduling } \\
\text { Pts progressing towards } \\
\text { surgery }\end{array}$ & $\begin{array}{l}\text { Scope of use } \\
\text { Timeliness }\end{array}$ & $\begin{array}{l}\text { Discrete event } \\
\text { simulation }\end{array}$ & $\begin{array}{l}\text { Pts awaiting cardiac } \\
\text { surgery at a tertiary } \\
\text { hospital in BC }\end{array}$ & 92 Pts & $\mathrm{P}, \mathrm{C}, \mathrm{T}$ & $\begin{array}{l}\text { Administrative } \\
\text { data }\end{array}$ & $\begin{array}{l}\text { WT1 (to apt) } \\
\text { WT2 (to surg) } \\
\text { Number of Pts } \\
\text { waiting for apts }\end{array}$ \\
\hline $\begin{array}{l}\text { Bungard et al } \\
(2008)^{26 /} \\
\text { Canada }\end{array}$ & $\begin{array}{l}\text { Single point-of-entry intake } \\
\text { and triage for tertiary care } \\
\text { and multidisciplinary clinic }\end{array}$ & Scope of use & Descriptive & $\begin{array}{l}\text { Cardiology } \\
\text { consultations in } \\
\text { Northern Alberta }\end{array}$ & NA & $\mathrm{P}, \mathrm{C}, \mathrm{T}$ & NA & $\rightarrow \mathrm{NA}$ \\
\hline
\end{tabular}




\begin{tabular}{|c|c|c|c|c|c|c|c|c|}
\hline $\begin{array}{l}\text { Author/year/ } \\
\text { country }\end{array}$ & Intervention & $\begin{array}{l}\text { Research } \\
\text { question } \\
\text { addressed }\end{array}$ & Study design & Sample source & Sample size & $\begin{array}{l}\text { Model of } \\
\text { single } \\
\text { entry }\end{array}$ & Data source & $\begin{array}{l}\text { Primary outcome } \\
\text { measures }\end{array}$ \\
\hline $\begin{array}{l}\text { Cipriano et al } \\
(2008)^{27 /} / \\
\text { Canada }\end{array}$ & $\begin{array}{l}\text { Discrete event simulation } \\
\text { to evaluate the effects } 4 \\
\text { waiting time management } \\
\text { strategies }\end{array}$ & $\begin{array}{l}\text { Scope of use } \\
\text { Timeliness }\end{array}$ & $\begin{array}{l}\text { Discrete event } \\
\text { simulation }\end{array}$ & $\begin{array}{l}\text { Ontario Joint } \\
\text { Replacement } \\
\text { Registry (Pts } \\
\text { awaiting hip or knee } \\
\text { replacement surgery) }\end{array}$ & 26583 Pts & $\mathrm{P}$ & $\begin{array}{l}\text { Administrative } \\
\text { data }\end{array}$ & $\begin{array}{l}\text { WT2-from } \\
\text { decision date to } \\
\text { undertake } \\
\text { surgery to date } \\
\text { of surgery- } \\
\text { tracked } \\
\text { regionally } \\
\text { Proportion of } \\
\text { Pts receiving } \\
\text { surgery within } \\
\text { benchmark }\end{array}$ \\
\hline $\begin{array}{l}\text { Bichel et al } \\
(2009)^{24} / \\
\text { Canada }\end{array}$ & $\begin{array}{l}\text { Central access and triage } \\
\text { processes across medical } \\
\text { specialties, prioritisation } \\
\text { tools, redesign of clinic } \\
\text { process flow }\end{array}$ & $\begin{array}{l}\text { Scope of use } \\
\text { Timeliness } \\
\text { Acceptability }\end{array}$ & $\begin{array}{l}\text { Post-test only } \\
\text { with non- } \\
\text { equivalent } \\
\text { groups }\end{array}$ & $\begin{array}{l}\text { Referrals for various } \\
\text { internal medicine } \\
\text { subspecialties in } \\
\text { Calgary, Alberta }\end{array}$ & Not reported & $\mathrm{P}, \mathrm{C}, \mathrm{T}$ & $\begin{array}{l}\text { Administrative } \\
\text { data }\end{array}$ & $\begin{array}{l}\text { WT1: time to } \\
\text { Apt } \\
\text { Acceptance of } \\
\text { referrals by } \\
\text { division (total } \\
\text { number) }\end{array}$ \\
\hline $\begin{array}{l}\text { Bungard et al } \\
(2009)^{25} / \\
\text { Canada }\end{array}$ & $\begin{array}{l}\text { Single point-of-entry intake } \\
\text { service and } \\
\text { multidisciplinary clinic }\end{array}$ & $\begin{array}{l}\text { Scope of use } \\
\text { Timeliness }\end{array}$ & $\begin{array}{l}\text { Simple pre/post } \\
\text { with non- } \\
\text { equivalent } \\
\text { groups }\end{array}$ & $\begin{array}{l}\text { Cardiology } \\
\text { consultations in } \\
\text { Northern Alberta }\end{array}$ & $\begin{array}{l}\text { Intervention: } \\
3096 \text { Pts } \\
\text { Control: } 311 \text { Pts }\end{array}$ & $\mathrm{P}, \mathrm{C}, \mathrm{T}$ & $\begin{array}{l}\text { Administrative } \\
\text { data }\end{array}$ & $\begin{array}{l}\text { WT1 (to initial } \\
\text { consultation) } \\
\text { WT2 (to } \\
\text { definitive final } \\
\text { diagnosis) } \\
\text { Number of new } \\
\text { referrals }\end{array}$ \\
\hline $\begin{array}{l}\text { Macleod et al } \\
(2009)^{28} / \\
\text { Canada }\end{array}$ & $\begin{array}{l}\text { Comprehensive model of } \\
\text { care: single wait list, } \\
\text { technology to support } \\
\text { referral management, } \\
\text { assessment services, } \\
\text { education, self- } \\
\text { management, treatment } \\
\text { programmes and specialist } \\
\text { care }\end{array}$ & $\begin{array}{l}\text { Scope of use } \\
\text { Timeliness }\end{array}$ & Descriptive & $\begin{array}{l}\text { Pts requiring hip or } \\
\text { knee replacement } \\
\text { surgery (Toronto } \\
\text { Central Local Health } \\
\text { Integration Network) }\end{array}$ & Not reported & $\mathrm{P}, \mathrm{C}, \mathrm{T}$ & $\begin{array}{l}\text { Administrative } \\
\text { data }\end{array}$ & $\begin{array}{l}\text { WT2-from } \\
\text { decision date to } \\
\text { undertake } \\
\text { surgery to date } \\
\text { of surgery }\end{array}$ \\
\hline $\begin{array}{l}\text { van den } \\
\text { Heuvel } \\
(2012)^{29} / \\
\text { Canada }\end{array}$ & $\begin{array}{l}\text { Hernia clinic based on a } \\
\text { group model of care: } \\
\text { centralised intake, triage } \\
\text { by surgeon, common } \\
\text { waiting list }\end{array}$ & $\begin{array}{l}\text { Scope of use } \\
\text { Timeliness } \\
\text { Acceptability }\end{array}$ & Cross-sectional & $\begin{array}{l}\text { Pts who had hernia } \\
\text { surgery at QEII in } \\
\text { Halifax, Nova Scotia }\end{array}$ & 94 Pts & $\mathrm{P}, \mathrm{C}, \mathrm{T}$ & Questionnaire & $\begin{array}{l}\text { WT1: from } \\
\text { referral to initial } \\
\text { consult } \\
\text { Pt acceptability }\end{array}$ \\
\hline
\end{tabular}


Table 2 Study results, conclusions and limitations

\begin{tabular}{|c|c|c|}
\hline Author & Results & Limitations \\
\hline $\begin{array}{l}\text { Ramchandani } \\
\text { et al }(2002)^{5}\end{array}$ & $\begin{array}{l}\text { Acceptability } \\
\text { Pts: } 82 \% \text { of Pts expressed willingness to change } \\
\text { consultants in order to get an earlier operation by a surgeon } \\
\text { of equal quality } \\
\text { GPs: } 92 \% \text { favoured pooled lists; } 8 \% \text { were against } \\
\text { Consultant ophthalmologists: } 30 \% \text { favoured (for routine } \\
\text { cases) and } 67 \% \text { were against pooled }\end{array}$ & $\begin{array}{l}\text { Small sample sizes for Pts and GPs } \\
\text { Low response consultant survey (64\%) } \\
\text {-views of responders may differ from } \\
\text { those of non-responders } \\
\text { (non-response bias) } \\
\text { Views of urban GPs may not reflect } \\
\text { those of rural GPs }\end{array}$ \\
\hline $\begin{array}{l}\text { Leach et al } \\
(2004)^{33}\end{array}$ & $\begin{array}{l}\text { Accessibility } \\
185 \text { days, reduced to } 30 \text { days following use of pooled lists } \\
\text { Before introduction of pooled waiting lists, } 37 \% \text { of Pts } \\
\text { waited for more than } 9 \text { months-this fell to } 0\end{array}$ & $\begin{array}{l}\text { Limited data presented } \\
\text { Source of preimplementation/ } \\
\text { postimplementation data not clear } \\
\text { Total number of Pts, WT1 and WT2 } \\
\text { data is not available }\end{array}$ \\
\hline $\begin{array}{l}\text { Singh et al } \\
(2005)^{31}\end{array}$ & $\begin{array}{l}\text { Accessibility } \\
\text { Waiting lists for the selected procedures were cleared, } \\
\text { especially longest waiters } \\
\text { Pt throughput improved; number of the selected surgical } \\
\text { procedures performed doubled } \\
\text { Cost: operating costs were reduced by } 25 \% \text { (largely due to } \\
\text { reduced length of stay); no recorded adverse Pt outcomes } \\
\text { Acceptability } \\
91 \% \text { of Pts felt the process was clearly explained to them; } \\
65 \% \text { felt a definite date of surgery was most important } \\
40 \% \text { did not mind that their consulting and operating } \\
\text { surgeons were different }\end{array}$ & $\begin{array}{l}\text { Small scale complex intervention with } \\
\text { many variables, difficult to assess } \\
\text { association between WT reduction and } \\
\text { use of single-entry components } \\
\text { No definitions or data provided for } \\
\text { waiting times } \\
\text { Comparison of groups from different } \\
\text { populations; survey sent to one group } \\
\text { (control group based on historical data) } \\
\text { Low questionnaire response rate-no } \\
\text { measure of overall satisfaction; } \\
\text { probable non-response bias }\end{array}$ \\
\hline $\begin{array}{l}\text { Sir-Ram et al } \\
(2005)^{32}\end{array}$ & $\begin{array}{l}\text { Accessibility } \\
\text { Mean total WT from referral to surgery in group } 1 \text { (direct } \\
\text { booking service) was } 70 \text { days (range } 10-177) \text {, much } \\
\text { shorter than for group } 2 \text { (control) } \\
\text { Group } 2 \text { mean WT1 was } 77 \text { days; WT2 } 84.2 \text { days, total WT } \\
161.2 \text { days ( } \mathrm{p}<0.05) \\
\text { Acceptability } \\
\text { 94\% of respondents would recommend the direct booking } \\
\text { service to a friend }\end{array}$ & $\begin{array}{l}\text { Survey only sent to one group (control } \\
\text { group was based on historical data) } \\
\text { Comparison of groups from different } \\
\text { populations } \\
\text { Low questionnaire response rate } \\
\text { Probable non-response bias }\end{array}$ \\
\hline $\begin{array}{l}\text { Vasilakis et al } \\
(2007)^{30}\end{array}$ & $\begin{array}{l}\text { Accessibility } \\
\text { Pooled lists reduced mean number of Pts waiting on the list } \\
\text { by } 30 \% \text {, compared with individual referrals } \\
\text { Twice as many Pts had appointments within } 12 \text { weeks of } \\
\text { referral through pooled vs individual surgeon referrals } \\
\text { Pooled referrals reduced WT1 among longest waiters } \\
\text { Pooled referrals increased WT2 for non-urgent cases; no } \\
\text { impact on urgent and semiurgent Pts or on total WT } \\
\text { Regardless of referral method, odds of surgery for Pts was } \\
\text { equal within } 18 \text { weeks }\end{array}$ & $\begin{array}{l}\text { Simulation models may not be true } \\
\text { representations of clinical scenarios } \\
\text { Unable to capture nuances of complex } \\
\text { interventions }\end{array}$ \\
\hline $\begin{array}{l}\text { Bungard et al } \\
(2008)^{26}\end{array}$ & $\begin{array}{l}\text { New collaborative model involves a single point-of-entry, } \\
\text { intake and triage mechanism with a multidisciplinary team to } \\
\text { ensure only one visit (rather than repeated) with cardiologist } \\
\text { Traditional referral patterns still respected }\end{array}$ & - NA \\
\hline $\begin{array}{l}\text { Cipriano et al } \\
(2008)^{27}\end{array}$ & $\begin{array}{l}\text { Accessibility } \\
\text { Clinically prioritising Pts reduced WTs for high-priority Pts } \\
\text { and increased the number of Pts in all priority levels } \\
\text { receiving surgery within maximum recommended WTs } \\
90 \% \text { of Pts received surgery within benchmark-achieved } \\
1 \text { year earlier } \\
\text { Efficiency/equity } \\
\text { Common waiting lists resulted in increased efficiency, equity } \\
\text { in WT across regions and reduced waiting times in the long } \\
\text { term } \\
\text { Regional variation in WTs was reduced }\end{array}$ & $\begin{array}{l}\text { Simulation models may not be true } \\
\text { representations of clinical scenarios } \\
\text { Reporting by surgeons to the OJRR is } \\
\text { voluntary therefore data may not be } \\
\text { fully representative }\end{array}$ \\
\hline
\end{tabular}


Table 2 Continued

\begin{tabular}{|c|c|c|}
\hline Author & Results & Limitations \\
\hline $\begin{array}{l}\text { Bichel et al } \\
(2009)^{24}\end{array}$ & $\begin{array}{l}\text { Accessibility } \\
\text { Participating clinics demonstrated varying results } \\
\text { - Centralised access and triage decreased WTs and enabled } \\
\text { timely access for Pts requiring urgent care (seen based on } \\
\text { urgency rather than for specific surgeon) } \\
\text { WTs decreased in spite of increased number of monthly Pt } \\
\text { referrals and acceptance rate for most clinics } \\
\text { WTs for consultation decreased from a mean }(S D) \text { of } 29 \\
( \pm 46) \text { to } 17( \pm 14) \text { days }(p<0.05) \text { for urgent-level referrals, } \\
\text { from } 110( \pm 57) \text { to } 63( \pm 42) \text { days }(p<0.00005) \text { for } \\
\text { moderate-level referrals, and from } 155( \pm 88) \text { to } 108( \pm 37) \\
\text { days for routine-level referrals, respectively, between } 2005 \\
\text { and } 2008 \\
\text { Efficiency: pooling of referrals eliminated duplicate } \\
\text { referralsEquity. WTs for each physician equalised }\end{array}$ & $\begin{array}{l}\text { Limited description of methods } \\
\text { employed } \\
\text { - Sampling technique, sample size not } \\
\text { provided } \\
\text { Preimplementation data (for } \\
\text { comparisons) was not available for all } \\
\text { groups }\end{array}$ \\
\hline $\begin{array}{l}\text { Bungard et al } \\
(2009)^{25}\end{array}$ & $\begin{array}{l}\text { Accessibility } \\
\text { Pts were seen significantly sooner in each year of Cardiac } \\
\text { EASE compared with pre-EASE period ( }<<0.0001) \\
\text { The mean WT from referral to specialist consultation (WT1) } \\
\text { was reduced from } 71 \pm 45 \text { days in the pre-EASE group to } 33 \\
\pm 19 \text { days in the EASE group ( } p<0.0001 \text { ) } \\
\text { Cardiac EASE Pts had a significantly shorter wait to } \\
\text { definitive diagnostic decision and treatment plan (WT2) } \\
\text { compared with pre-EASE ( } 51 \pm \text { days and } 120 \pm 86 \text { days, } \\
\text { respectively) } \\
\text { Increased Pt volume through Cardiac EASE ( } 50 \% \text { from } \\
2004 \text { to } 2005 ; 19 \% \text { from } 2005 \text { to } 2006 \text { ) }\end{array}$ & $\begin{array}{l}\text { Complex intervention with many } \\
\text { variables, difficult to assess } \\
\text { association between WT reduction and } \\
\text { use of single-entry components } \\
\text { Comparison of groups from different } \\
\text { populations } \\
\text { Historical group has a small sample } \\
\text { size compared with that of the } \\
\text { intervention group } \\
\text { Treatment effect may be present }\end{array}$ \\
\hline $\begin{array}{l}\text { Macleod et al } \\
(2009)^{28}\end{array}$ & $\begin{array}{l}\text { Accessibility } \\
\text { - } 90 \% \text { of Pts waited }<115 \text { days for hip or knee replacement } \\
\text { surgery (WT2; less than provincial target of } 182 \text { days); WT1 } \\
\text { was }<100 \text { days }\end{array}$ & $\begin{array}{l}\text { Little empirical evidence for results } \\
\text { cited } \\
\text { No comparison provided to specific } \\
\text { previous WTs/scenarios }\end{array}$ \\
\hline $\begin{array}{l}\text { van den Heuvel } \\
(2012)^{29}\end{array}$ & $\begin{array}{l}\text { Accessibility } \\
\text { 94/236 ( } 40 \% \text { ) Pts responded-67\% had the same surgeon } \\
\text { for assessment and surgery; } 31 \% \text { had a different surgeon } \\
\text { (next-available) } \\
\text { Almost half of respondents ( } 48 \% \text { ) did not understand that } \\
\text { choosing a specific surgeon may result in longer waiting } \\
\text { times } \\
\text { No difference in postoperative complication rates between } \\
\text { groups } \\
\text { WTs from referral to initial consult in the hernia clinic (WT1) } \\
\text { decreased from } 208 \text { to } 59 \text { days ( } 2007-2009 \text { ) } \\
\text { Acceptability } \\
\text { Two thirds of Pts had confidence in the competence of any } \\
\text { surgeon and were comfortable having their surgery } \\
\text { performed by a surgeon they meet on the day of surgery } \\
\text { - Even if Pts have a different surgeon for their operation than } \\
\text { for their assessment, their confidence is high ( } 86.2 \% \text { ) } \\
\text { Most Pts felts that service is faster and better in a } \\
\text { specialised centre (like the hernia clinic being evaluated) }\end{array}$ & $\begin{array}{l}\text { Low questionnaire response rate- } \\
\text { results may not be generalisable } \\
\text { Probable non-response bias }\end{array}$ \\
\hline
\end{tabular}

EASE, Ensuring Access and Speedy Evaluation; GP, general practitioner; NA, not available; OJRR, Ontario Joint Replacement Registry; Pt, patient; WT, waiting time; WT1, time from referral to initial consult; WT2, time from consult to procedure date.

length between providers (equalisation), leading to greater equity of access within the system of care. ${ }^{24} 2733$ Given the variability between study designs and scope, and generally focused environments within which interventions were introduced, the apparent improvements in 'efficiency' and 'equity of access' attributable to SEMs may not necessarily be generalisable.

Costs were reported in only one study-Singh and colleagues cite an overall cost-savings but this refers to savings accrued across the entire continuum of care 
Table 3 Assessment of study quality (selective items reported; derived from an adapted Downs and Black checklist; ${ }^{21}$ higher score indicates better study quality)

\begin{tabular}{|c|c|c|c|c|c|c|c|c|c|c|c|c|}
\hline Author & Data source & $\begin{array}{l}\text { Study } \\
\text { population } \\
\text { described }\end{array}$ & $\begin{array}{l}\text { Ethics } \\
\text { approval } \\
\text { mentioned }\end{array}$ & $\begin{array}{l}\text { Sample } \\
\text { size } \\
\text { explained }\end{array}$ & $\begin{array}{l}\text { Standard } \\
\text { of care } \\
\text { described }\end{array}$ & $\begin{array}{l}\text { Intervention } \\
\text { described }\end{array}$ & $\begin{array}{l}\text { Waiting } \\
\text { time } \\
\text { definitions } \\
\text { provided }\end{array}$ & $\begin{array}{l}\text { Control } \\
\text { group } \\
\text { present }\end{array}$ & $\begin{array}{l}\text { Baseline } \\
\text { group } \\
\text { differences } \\
\text { discussed }\end{array}$ & $\begin{array}{l}\text { Statistical } \\
\text { tests } \\
\text { used }\end{array}$ & $\begin{array}{l}\text { Limitations } \\
\text { discussed }\end{array}$ & $\begin{array}{l}\text { Downs } \\
\text { and black } \\
\text { quality } \\
\text { score } \\
\text { (/21) }\end{array}$ \\
\hline $\begin{array}{l}\text { Ramchandani } \\
\text { et al (2002) }\end{array}$ & $\begin{array}{l}\text { Questionnaire } \\
\text { Interview }\end{array}$ & Yes & No & Yes & Yes & Yes & Yes & No & No & No & Yes & 17 \\
\hline $\begin{array}{l}\text { Leach et al } \\
(2004)^{33}\end{array}$ & $\begin{array}{l}\text { Administrative } \\
\text { data }\end{array}$ & Yes & No & No & No & Yes & Yes & Yes & No & No & Yes & 10 \\
\hline $\begin{array}{l}\text { Singh et al } \\
(2005)^{31}\end{array}$ & $\begin{array}{l}\text { Administrative } \\
\text { data }\end{array}$ & Yes & Yes & Yes & Yes & Yes & No & Yes & No & Yes & Yes & 9 \\
\hline $\begin{array}{l}\text { Sri-Ram et al } \\
(2005)^{32}\end{array}$ & Hospital data & Yes & No & Yes & Yes & Yes & Yes & Yes & Yes & Yes & Yes & 8 \\
\hline $\begin{array}{l}\text { Vasilakis et al } \\
(2007)^{30}\end{array}$ & $\begin{array}{l}\text { Administrative } \\
\text { data }\end{array}$ & Yes & No & Yes & Yes & Yes & Yes & Yes & No & Yes & Yes & 21 \\
\hline $\begin{array}{l}\text { Bungard et al } \\
(2008)^{26}\end{array}$ & Descriptive & Yes & No & NA & Yes & Yes & Yes & NA & NA & NA & NA & 17 \\
\hline $\begin{array}{l}\text { Cipriano et al } \\
(2008)^{27}\end{array}$ & $\begin{array}{l}\text { Administrative } \\
\text { data }\end{array}$ & Yes & No & Yes & Yes & Yes & Yes & Yes & No & Yes & Yes & 14 \\
\hline $\begin{array}{l}\text { Bichel et al } \\
(2009)^{24}\end{array}$ & $\begin{array}{l}\text { Administrative } \\
\text { data }\end{array}$ & Yes & No & No & No & Yes & Yes & Yes & No & Yes & Yes & 13 \\
\hline $\begin{array}{l}\text { Bungard et al } \\
(2009)^{25}\end{array}$ & $\begin{array}{l}\text { Administrative } \\
\text { data }\end{array}$ & Yes & Yes & Yes & Yes & Yes & Yes & Yes & Yes & Yes & Yes & 20 \\
\hline $\begin{array}{l}\text { Macleod et al } \\
(2009)^{28}\end{array}$ & $\begin{array}{l}\text { Administrative } \\
\text { data }\end{array}$ & Yes & No & No & Yes & Yes & Yes & Yes & No & No & Yes & 11 \\
\hline $\begin{array}{l}\text { van den } \\
\text { Heuvel } \\
(2012)^{29}\end{array}$ & Questionnaire & Yes & No & Yes & Yes & Yes & Yes & Yes & No & Yes & Yes & 18 \\
\hline
\end{tabular}




\begin{tabular}{|c|c|c|c|c|c|c|c|c|c|c|c|c|c|}
\hline & & & Accessib & bility & & & Acce & eptabil & ility & & & & \\
\hline Author & $\begin{array}{l}\text { Clinical } \\
\text { area }\end{array}$ & $\begin{array}{l}\text { Model of } \\
\text { single } \\
\text { entry }\end{array}$ & $\begin{array}{l}\text { Waiting } \\
\text { list } \\
\text { length } \\
\end{array}$ & $\begin{array}{l}\text { Waiting } \\
\text { time }\end{array}$ & $\begin{array}{l}\text { Proportion meeting } \\
\text { benchmark }\end{array}$ & $\begin{array}{l}\mathrm{Pt} \\
\text { volume }\end{array}$ & $\begin{array}{l}\text { Pt } \\
\text { Sat }\end{array}$ & $\begin{array}{l}\text { Phys } \\
\text { Sat }\end{array}$ & $\begin{array}{l}\text { Surgeon } \\
\text { Satisfaction }\end{array}$ & Efficiency & Equity & Cost & Appropriateness \\
\hline $\begin{array}{l}\text { Ramchandani } \\
\text { et al }(2002)^{5}\end{array}$ & Cataract surg & $P$ & NR & NR & $\begin{array}{l}\text { NR (WT1: } 3 \text { mos) } \\
\text { (WT2: } 6 \text { mos) }\end{array}$ & NR & + & + & - & NR & NR & NR & NR \\
\hline $\begin{array}{l}\text { Cipriano et al } \\
(2008)^{27}\end{array}$ & $\begin{array}{l}\text { Hip and knee } \\
\text { replacement }\end{array}$ & P & NR & $\downarrow$ WT2 & $\begin{array}{l}\uparrow \text { for surg (WT2: } \\
6 \mathrm{mos} \text { ) }\end{array}$ & NR & NR & NR & NR & $\uparrow$ & $\uparrow$ & NR & NR \\
\hline $\begin{array}{l}\text { Leach et al } \\
(2004)^{33}\end{array}$ & Neurosurgery & $\mathrm{P}, \mathrm{C}, \mathrm{T}$ & $\downarrow$ & $\downarrow$ TW & $\begin{array}{l}\uparrow \text { for apt+surg (WT1: } \\
3 \text { mos) (WT2: } 6 \text { mos) }\end{array}$ & NR & + & NR & NR & NR & $\uparrow$ & NR & $\uparrow$ \\
\hline $\begin{array}{l}\text { Singh et al } \\
(2005)^{31}\end{array}$ & Various elective & $\mathrm{P}, \mathrm{C}, \mathrm{T}$ & $\downarrow$ & $\downarrow$ TW & NR & $\uparrow$ & + & NR & NR & $\uparrow$ & NR & $\downarrow$ & NR \\
\hline $\begin{array}{l}\text { Sri-Ram et al } \\
(2005)^{32}\end{array}$ & Hernia & $\mathrm{P}, \mathrm{C}, \mathrm{T}$ & $\downarrow$ & $\downarrow \mathrm{TW}$ & $\begin{array}{l}\uparrow \text { for surg (WT1: } \\
3 \text { mos) (WT2: } 6 \text { mos) }\end{array}$ & NR & + & NR & NR & $\uparrow$ & NR & NR & $\uparrow$ \\
\hline $\begin{array}{l}\text { Vasilakis et al } \\
(2007)^{30}\end{array}$ & Cardiac surg & $\mathrm{P}, \mathrm{C}, \mathrm{T}$ & $\downarrow$ & $\downarrow$ WT1 & $\begin{array}{l}\uparrow \text { for apt; varied for } \\
\text { surg by priority (12/ } \\
18 \text { weeks) }\end{array}$ & NR & NR & NR & NR & NR & NR & NR & NR \\
\hline $\begin{array}{l}\text { Bungard et al } \\
(2008)^{26}\end{array}$ & $\begin{array}{l}\text { Cardiac } \\
\text { consultation }\end{array}$ & $P, C, T$ & NR & NR & NR & NR & $\mathrm{NR}$ & NR & NR & NR & NR & NR & NR \\
\hline $\begin{array}{l}\text { Bichel et al } \\
(2009)^{24}\end{array}$ & $\begin{array}{l}\text { Various internal } \\
\text { medicine } \\
\text { subspecialties }\end{array}$ & $\mathrm{P}, \mathrm{C}, \mathrm{T}$ & NR & $\downarrow$ WT1 & NR & $\uparrow$ & NR & NR & NR & $\uparrow$ & $\uparrow$ & NR & $\uparrow$ \\
\hline $\begin{array}{l}\text { Bungard et al } \\
(2009)^{25}\end{array}$ & $\begin{array}{l}\text { Cardiac } \\
\text { consultation }\end{array}$ & $P, C, T$ & NR & $\begin{array}{l}\downarrow \text { WT1 } \\
\downarrow \text { WT2 }\end{array}$ & $\begin{array}{l}\uparrow \text { for apt (WT1: } \\
4-6 \text { weeks) }\end{array}$ & $\uparrow$ & $N R$ & NR & NR & $\uparrow$ & NR & NR & $\uparrow$ \\
\hline $\begin{array}{l}\text { Macleod et al } \\
(2009)^{28}\end{array}$ & $\begin{array}{l}\text { Hip and knee } \\
\text { replacement }\end{array}$ & $P, C, T$ & NR & $\downarrow$ WT2 & $\begin{array}{l}\uparrow \text { for surg (WT2: } \\
6 \text { mos) }\end{array}$ & NR & + & NR & NR & NR & NR & NR & $\uparrow$ \\
\hline $\begin{array}{l}\text { van den Heuvel } \\
\text { et al }(2012)^{29}\end{array}$ & Hernia (multiple) & $\mathrm{P}, \mathrm{C}, \mathrm{T}$ & NR & $\downarrow$ WT1 & $\mathrm{NR}$ & NR & + & NR & NR & $\uparrow$ & NR & NR & NR \\
\hline
\end{tabular}


(from mostly reductions in length of stay). Among several concurrent variables and interventions, it is difficult to say how much of the savings are associated with the use of components of single entry. ${ }^{31}$ Bungard et $a 2^{25}$ described the Cardiac EASE programme as 'relatively inexpensive'.

\section{Influence of SEMs on patient-centredness of elective services}

Patient acceptability

Although only two studies explicitly reported patient acceptability of SEMs, ${ }^{5}{ }^{32}$ six studies reported positive patient satisfaction with SEMs. ${ }^{5} 2829$ 31-33 Patient surveys generally had small sample sizes and low response rates, increasing the likelihood of non-response bias. ${ }^{5} 293132$

Patient responses indicated that waiting times resulting from the implementation of new programmes were more agreeable; patient satisfaction was generally high. Though many patients expressed a desire to remain with their specific chosen surgeon, there was a willingness to see the next-available surgeon (if lists are pooled, and provided that the surgeons were equally qualified and that waiting times would decrease). ${ }^{5}$ One study found that although patients had expressed a desire to have the consulting and operating surgeons be the same, once it is the case that the operating surgeon is different, they no longer consider it important and express a high level of confidence in the operating surgeon. ${ }^{29}$ Reassurance, and proper explanations also contributed to patient acceptance and recommendation to others of new programmes. ${ }^{29} 3133$

\section{Physician/surgeon acceptability}

Only one study reported physician and/or surgeon satisfaction with SEMs ${ }^{5}$ finding that GPs favoured the notion of pooling lists more so than consultants. GPs also favoured pooling if it meant a reduction in waiting times and an equally experienced surgeon sees patients. Ramchandani et a $a \bar{p}$ reported that some surgeons felt that 'pooled lists were suitable only for routine cases'. Both GPs and consultants cited 'loss of responsibility for care, devaluation of the doctor-patient relationship and loss of consultant control' as among their reasons for opposing pooled lists. ${ }^{5}$ Consultants felt that they should complete the entire treatment; especially once they have seen a patient, rather than portions thereof and behave like a 'technician on a production line'. No study reported universal specialist/surgeon participation/compliance.

\section{DISCUSSION}

\section{Summary}

The aim of this review was to (1) summarise existing research on the scope, use and implementation of SEMs for elective surgical services; (2) to report on the evidence about the influence of SEMs on timeliness and access; and (3) patient-centredness (patient and provider acceptability) of SEMs. Eleven studies of various elective procedures were included from Canada, UK and Australia. Studies were of generally low rigour and weak observational design-two involved pooled waiting lists only, ${ }^{527}$ the remaining nine employed a combination of pooled lists, centralised intake and triage. Studies (including two simulation) showed that where used, SEMs showed a decrease in patient waiting times, ${ }^{24} 25{ }^{27-33}$ an increased proportion of individuals were meeting clinically recommended benchmark waiting times 252728303233 and a decrease in waiting list length $^{27}{ }^{30-33}$ when compared with a control group or to historic experience of controls. Patient acceptability, where reported, suggested high levels of satisfaction. $^{5} 282931-33$ Acceptability among GPs/surgeons was mixed. ${ }^{5}$

\section{Limitations}

Very few studies evaluated the influence of SEMs on timeliness and patient-centredness. Methods were limited or poorly described in most studies; rigour was low. While the Downs and Black checklist helped quantify this, the scores may not reflect this entirely. The tool is widely used and recommended, has been assessed to be valid and reliable but has scored low for external validity (on the external validity subscales for internal consistency reliability, inter-rater reliability). ${ }^{22}$ Issues with confounding and generalisability were consistent and studies varied substantially in design, scope, outcomes and reporting. Sample sizes were small and comparison groups were not equal or poorly compared. With several concurrent interventions at play (longer working hours, increased staff and incentives) and with little adjustment for covariates, one cannot determine the extent to which observed decreases in waiting times are attributable to SEMs. Complexity and contextual factors (ie, referral management processes, clinic/hospital capacity, participation rates, scope and scale) also varied from study to study. ${ }^{24}$

Reporting of only WT1 or WT2 gives an incomplete representation of changes to patient waiting times and further makes comparison across studies challenging. Without the combined waiting time, we cannot be sure if waiting times were reduced or merely shifted (ie, WT1 reduced, but WT2 increases). Reporting of only total waiting time makes it difficult to identify the sources of the improvement within the referral process. Finally, causality cannot be proven or established; it cannot be said that waiting times decrease definitively through the use of SEMs. The ideal way to establish causality is through the use of randomised experiments. Randomised controlled trials, while seen as the gold standards of evidence for the effectiveness of interventions ${ }^{34}$ may neither be possible nor appropriate for the study of SEMs. However, high-quality and rigourous, controlled observational studies are lacking and such studies would strengthen the quality and reliability of further research.

The lack of methodologically sound evaluation studies on SEMs may be because conducting research in this 
area is difficult (ie, experimental design, limited use of SEMs, limited willingness to participate). We included two discrete event simulation studies, modelling components of SEMs, whose findings were consistent with studies of other designs. Simulation models, however, may not be true representations of clinical scenarios and may not be able to capture nuances of complex interventions. No included study demonstrated unfavourable results, or evidence that would indicate that SEMs are ineffective in reducing waiting times. Publication bias may be important to consider. Finally, we did not review the grey literature.

\section{What our review adds to the literature}

Our review is the first to examine and summarise the influence of SEMs on access to adult elective surgical services, their influence on patient flow and waiting times for elective services and acceptability of SEMs to patients and providers (GPs and surgeons).

A 2015 Cochrane systematic review assessed 'the effectiveness of interventions aimed at reducing waiting times for elective care'. Of the eight studies included and five interventions assessed, only one was a SEM (also included in our review). ${ }^{1133}$ The results, concern with quality, conclusions and recommendations were similar to those in our review, but were based on assessments of multiple waiting time management strategies. Our review focused solely on SEMs and therefore provides a more thorough assessment of the available, related literature.

SEMs appear to be most effective if employed in concert and accompanied by additional resources, such as multidisciplinary preassessment. Availability of resources and careful policy planning (ie, use of waiting time management strategies) to develop capacity is important to consider within a universal, publicly funded health system as demand grows. ${ }^{1} 935$ If not addressed adequately, waiting time concerns could lead to fewer patients meeting clinically recommended benchmarks, poorer experience and clinical outcomes. ${ }^{76-39}$

Our study also reveals the importance of patient, physician and surgeon acceptability and cooperation. While promising, the adoption of any waiting time management strategy will require the support of several stakeholders. Knowing that waiting times will decrease will increase acceptability but there will also be those who will not accept SEMs under any circumstances, and will be comfortable with their preferences and resultant willingness to wait. Recent related studies in the literature have shown that some patients (typically older) awaiting surgery may prefer to see a surgeon of their choice (over the next-available), citing trust, surgeon reputation and a willingness to wait longer. ${ }^{40-43}$

\section{Future research}

Additional studies are needed which explore the acceptability of all related stakeholders that would be affected by SEMs, including family members, GPs, surgeons, office assistants and decision-makers. Their viewpoints are of tremendous importance given the diverse roles they play on the continuum of care for elective surgical services. In order to improve accessibility, uptake of SEMs needs to increase among all stakeholders. Patients need to be informed and reassured and both physician and surgeon suggestions must be considered when planning, designing and implementing SEMs. If SEMs were to gain wider acceptance and use, it will also be critical that unanticipated consequences and implications for efficiency, equity and cost are further evaluated, especially as the need for demonstrated cost-effective strategies is critical within publicly funded health systems with limited resources.

Additional studies could consider the influence of SEMs and reduced waiting times on surgical outcomes and the other dimensions of quality. Best practice surrounding their design and implementation could also be profiled. Future research could employ an interrupted time series design, with multiple data points before and after the intervention to determine the contribution of SEMs to waiting time reduction. Unanticipated consequences and critical success factors such as physician payment schemes, administration, resource availability, individual stakeholder roles along the care continuum could provide valuable tools and insight for increasing the use and acceptability of SEMs.

\section{CONCLUSION}

The small number of low-quality studies available makes it challenging to draw firm conclusions about the effectiveness of SEMs in improving timeliness of access to elective procedures. Our findings show a consistently positive impact by SEMs on the access-related variables. While promising, they also prompt the need for ongoing study in critical areas, but with higher quality designs, more comprehensive scope and greater methodological rigour.

Acknowledgements The authors thank Diane Lorenzetti for her assistance in developing our search strategy and Paul Ronksley for his support of our project.

Contributors ZD initiated and contributed to the design of the collaborative project; collected and analysed the data; drafted, revised and finalised the draft paper and is also the guarantor. BC-S designed data collection tools; collected, analysed and monitored data collection and revised the draft paper. TN contributed to the design of the collaborative project; collected and analysed the data; drafted and revised the draft paper. HTS contributed to the design of the collaborative project; monitored data collection and revised the draft paper. TWN initiated the collaborative project, monitored data collection and analysis and revised the draft paper. DAM contributed to the design of the collaborative project, monitored data collection and revised the paper. All authors approved the final version of the paper for submission.

Funding This research was supported by CIHR Emerging Team Grant 92252.

Competing interests TN reports that she is the programme facilitator for Alberta Health Services. DAM reports other from Canada Research Chair, Health Services and Systems Research, other from Arthur J.E. Child Chair in Rheumatology Outcomes Research, during the conduct of the study; personal fees from Abbvie, personal fees from Janssen, personal fees from Novartis, 
personal fees from Optum Insight, outside the submitted work. The content (in part) was prepared for a poster presentation by ZD at the 35th Annual North American Meeting of the Society for Medical Decision Making (SMDM); Baltimore, MD: 23 October 2013. The abstract was subsequently published as follows: Damani Z, Conner-Spady B, Noseworthy T. P4-39 Value and Acceptability of Single-Entry Models in Health Care (Abstract). Med Decis Making. February 2014;34(2):E129.

Provenance and peer review Not commissioned; externally peer reviewed.

Data sharing statement As this is a systematic review, all data extracted for the source articles in the literature are contained within our data tables.

Open Access This is an Open Access article distributed in accordance with the Creative Commons Attribution Non Commercial (CC BY-NC 4.0) license, which permits others to distribute, remix, adapt, build upon this work noncommercially, and license their derivative works on different terms, provided the original work is properly cited and the use is non-commercial. See: http:// creativecommons.org/licenses/by-nc/4.0/

\section{REFERENCES}

1. Waiting Time Policies in the Health Sector. What works? In: Sicilian $\mathrm{L}$, Borowitz M, Moran V, eds. OECD health policy studies. OECD Publishing, 2013

2. Harrison A, New B. Access to elective care. What should really be done about waiting lists. London: King's Fund Publishing, 2000

3. Mossialos E, Wenzl M, Osborn R, et al. International profiles of health care systems, 2014. The Commonwealth Fund, 2015.

4. Kreindler SA. Watching your wait: evidence-informed strategies for reducing health care wait times. Qual Manag Health Care 2008;17:128-35.

5. Ramchandani M, Mirza S, Sharma A, et al. Pooled cataract waiting lists: views of hospital consultants, general practitioners and patients. J R Soc Med 2002;95:598-600.

6. Akbari A, Mayhew A, Al-Alawi MA, et al. Interventions to improve outpatient referrals from primary care to secondary care. Cochrane Database Syst Rev 2008;(4):CD005471.

7. Kreindler SA. Policy strategies to reduce waits for elective care: a synthesis of international evidence. Br Med Bull 2010;95 7-32.

8. Bodenheimer T. Coordinating care-a perilous journey through the health care system. N Engl J Med 2008;358:1064-71.

9. Siciliani L, Moran V, Borowitz M. Measures and comparing health care waiting times in OECD countries. Health Policy 2014;118:292-303.

10. Imison C, Naylor C. Referral Management - lessons for success. London: The King's Fund, 2010. http://www.kingsfund.org.uk/ publications/referral management.html

11. Ballini L, Negro A, Maltoni S, et al. Interventions to reduce waiting times for elective procedures. Cochrane Database Syst Rev 2015(2): CD005610.

12. Wait Times for Priority Procedures in Canada, 2014. Canadian Institute for Health Information, 2014.

13. Palvannan RK, Teow KL. Queueing for healthcare. J Med Syst 2012;36:541-7

14. Novak K, Veldhuyzen Van Zanten S, Pendharkar SR. Improving access in gastroenterology: the single point of entry model for referrals. Can J Gastroenterol 2013;27:633-5.

15. Alberta Hip and Knee Replacement Pilot Project. Scientific Evaluation Report. Calgary, AB: Alberta Bone and Joint Health Institute, 2007.

16. Priest A, Rachlis M, Cohen M. Why wait? Public solutions to cure surgical waitlists. A submission to the $B C$ government's conversation on health. Vancouver, BC: Canadian Centre for Policy Alternatives, 2007.

17. Institute of Medicine: envisioning the National Health Care Quality Report. MP Hurtado, EK Swift, JM Corrigan, Eds. Washington DC National Academy Press, 2001.

18. The Alberta Quality Matrix for Health. Health Quality Council of Alberta, 2016. http://hqca.ca/about/how-we-work/the-alberta-qualitymatrix-for-health-1

19. Popay J, Roberts H, Sowden A, et al. Guidance on the conduct of narrative synthesis in systematic reviews: a product from the ESRC methods programme. ERC methods program. Lancaster, PA: Lancaster University, 2006.
20. Moher D, Liberati A, Tetzlaff J, et al. Preferred reporting items for systematic reviews and meta-analyses: the PRISMA statement. PLoS Med 2009;6:e1000097.

21. Downs $\mathrm{SH}$, Black N. The feasibility of creating a checklist for the assessment of the methodological quality both of randomised and non-randomised studies of health care interventions. J Epidemiol Community Health 1998;52:377-84.

22. Quality checklist for health care intervention studies. Hamilton, ON McMaster Universiy, 2008 (11 September 2016). http://www.nccmt. $\mathrm{ca} /$ resources/search/9

23. Viswanathan M, Ansari M, Berkman N, et al. Assessing the risk of bias of individual studies in systematic reviews of health care interventions. Agency for Healthcare Research and Quality Methods Guide for Comparative Effectiveness Reviews, 2012.

24. Bichel A, Erfle S, Wiebe V, et al. Improving patient access to medical services: preventing the patient from being lost in translation. Healthc Q 2009;13:61-8.

25. Bungard TJ, Smigorowsky MJ, Lalonde LD, et al. Cardiac EASE (Ensuring Access and Speedy Evaluation)-the impact of a single-point-of-entry multidisciplinary outpatient cardiology consultation program on wait times in Canada. Can J Cardio 2009;25:697-702.

26. Bungard TJ, Smigorowsky MJ, Lalonde LD, et al. Cardiac EASE (Ensuring Access and Speedy Evaluation)—design of a single point of entry and a multidisciplinary team to reduce waiting times in the Canadian health care system. Healthc Manage Forum 2008;21:35-40

27. Cipriano LE, Chesworth BM, Anderson CK, et al. An evaluation of strategies to reduce waiting times for total joint replacement in Ontario. Med Care 2008;46:1177-83.

28. MacLeod AM, Gollish J, Kennedy D, et al. Towards a joint health and disease management program. Toronto hospitals partner to provide system leadership. Healthc Q 2009;12:56-65.

29. van den Heuvel B, Vair B, Porter G, et al. Patient compliance with a group model of care: the hernia clinic. Can J Surg 2012;55: 259-63.

30. Vasilakis C, Sobolev B, Kuramoto L, et al. A simulation study of scheduling clinic appointments in surgical care: individual surgeon versus pooled lists. J Operational Res Soc 2007;58:202-11.

31. Singh N, Brooke-Cowden GL, Whitehurst C, et al. The Auburn Elective Surgery Pilot Project. ANZ J Surg 2005;75:768-75.

32. Sriram K, Irvine T, InghamClark CL. A Direct Booking Hernia Service - a shorter wait and a satisfied patient. J Ambul Surg 2006;12:113-17.

33. Leach $\mathrm{P}$, Rutherford SA, King AT, et al. Generic waiting lists for routine spinal surgery. J R Soc Med 2004;97:119-20.

34. Rossi PH, Freeman HE. Evaluation a systematic approach. Newbury Park, CA: SAGE Publications, 1993.

35. Masri BA, Cochrane N, Duncan C, et al. Priority criteria for hip and knee replacement: addressing health service wait times. Report II Inventory of Initiatives Joint Replacement: International Approaches to Meeting the Needs. University of British Columbia.

36. Hoogeboom TJ, van den Ende $\mathrm{CH}$, van der Sluis $\mathrm{G}$. The impact of waiting for total joint replacement on pain and functional status: a systematic review. Osteoarthritis Cartilage 2009;17:1420-7.

37. Snider MG, MacDonald SJ, Pototschnik R. Waiting times and patien perspectives for total hip and knee arthroplasty in rural and urban Ontario. Can J Surg 2005;48:355-60.

38. Hajat S, Fitzpatrick R, Morris R, et al. Does waiting for total hip replacement matter? Prospective cohort study. J Health Serv Res Policy 2002;7:19-25.

39. Jones CA, Voaklander DC, Johnston DW, et al. The effect of age on pain, function, and quality of life after total hip and knee arthroplasty. Arch Intern Med 2001;161:454-60.

40. Birk HO, Henriksen LO. Why do not all hip and knee patients facing long waiting times accept re-referral to hospitals with short waiting time? Questionnaire study. Health Policy 2006;77:318-25.

41. Conner-Spady B, Marshall D, Bohm E, et al. Eliciting patient views on choosing the next available surgeon to reduce waiting times for joint replacement surgery: on the need to consider individual patient preferences and information needs. Int $J$ Person Centred Med 2011;1:362-8.

42. Connor-Spady BL, Johnston GH, Sanmartin C, et al., Saskatchewan Surgical Care Network/Western Canada Waiting List Project Research and Evaluation Working Group Committee. A bird can't fly on one wing: patient views on waiting for hip and knee replacement surgery. Health Expect 2007;10:108-16.

43. Conner-Spady B, Sanmartin C, Johnston G, et al. Willingness of patients to change surgeons for a shorter waiting time for joint arthroplasty. CMAJ 2008;179:327-32. 